\title{
Application and Analysis of BIM Technology in Chinese Architectural Design Industry
}

\author{
Chunming Zhang \\ Yunnan Arts University, Yunnan, 650500
}

Keywords: architectural engineering; quality control; architectural design

\begin{abstract}
More than 20 years ago, in the domestic engineering design industry, a technical revolution called CAD (Computer-Aided Design) announced that the engineering design industry began to bid farewell to the traditional "drawing board + T-ruler" manual drawing mode; Nowadays, another wave of technology, BIM (Building Information Modeling), has emerged as a wave of technology, and it has gradually swept at home and abroad and from the north to the south. With the popularization and penetration of the BIM technology revolution, it will end the "drawing era" of the engineering design industry and usher in a new "model era"; the construction engineers and technicians no longer leaned over to look at the engineering blueprints. The scenes where a person's hands are working on an iPad to manage operations and direct operations may not be far away.
\end{abstract}

\section{Introduction}

It is precisely as a result of this process that the concept of BIM is increasingly recognized and approved in the construction industry, and its role in the field of architectural design is also increasingly apparent. As a crucial design stage in the construction project life cycle, the use of BIM will play a more important role in the production of architectural design companies. In the future, how will the informatization of the construction industry promote the construction towards a more all-inclusive direction? What is the direction and trend of its informatization? With the rapid development of urban modernization, from the future construction industry to various companies, both the level of management and technical standards will complete the transformation of their own informatization in this innovation. In this process, the architectural design undoubtedly provides the most solid foundation support for the data building!

As is known to all, in the design phase, BIM can effectively integrate superior resources, accurately express intents, and reduce design errors, it depends on BIM technology and allows the project team to make changes or modifications to the project anytime anywhere in the engineering design or documentation process. Because once modified, the result of the modification will be displayed in real time and automatically coordinated in all fields and links of the whole project. That is, the BIM 3D engineering model can automatically coordinate the improper expression and omission of 2D drawings, eliminating the need for heavy, low-value repeated coordination and manual inspection links, improving inspection communication efficiency, accurately communicating designer intent, and further improving overall work quality. This also allows the project team to devote more time and effort to the more pivotal and critical issues of the project.

As the advantages of BIM in the design industry are increasingly evident, it can be foreseen that the information management of architectural design will undoubtedly become the only way for traditional industries to obtain new life. Making full use of the huge productivity brought by information technology and improving the level of information application and management should become the most important part of the competitiveness of the architectural design industry. Although at present, more and more new technologies, such as BIM and cloud computing mobile applications, are flooding the informationization of construction companies, however, Yuan Zhenggang pointed out sharply: "At present, informatization presents some problems such as faults and isolated islands of high level attention, middle level shortsightedness, basic level neglect, and some problems in informatization, such as insufficient investment, lack of standardization, lack of standards, and so on.” As Zhang Jianping said: "We now plan to integrate the existing information 
technology of BIM and enterprise projects, but now everyone has a little misunderstanding in concept: Is a three-dimensional model BIM? A three-dimensional model is certainly not BIM. It does not reflect processes and functions. The three elements of a model are the product model, the process model, and the decision model. It is a combination.” In other words, the concept of BIM will only become clear after the standard has been consolidated.

\section{Quantification and Qualitative Division of BIM Design Stage}

As mentioned above, only under the guidance of a unified industry standard, the efficiency of BIM's application in the architectural design industry can be well achieved, therefore, the formulation of a unified standard has become an urgent matter for the current acceleration of the development of BIM in the architectural design industry. For the BIM process in architectural design, through the practice summary, we propose such a process scenario and specific technical details:

As a design project according to BIM requirements, the first is to define a recognized design implementation process. Under such a process, according to the different stages of the design, the proposed digital model proposes specific and coherent design technical standards and processes at different stages. In addition, the general responsible person for the design compiles and collects periodical results to submit to the owner for review and adjustment, and has ensured the harmonization of the work standards and the coordination of operations, the details are as follows:

\subsection{Schematic design phase:}

The architectural design program uses REVIT software to digitally design the solution model. Finally, the chief designer integrates the individual digital models, at this stage, the model focuses on the body shape and space analysis.

\subsection{Preliminary design phase}

When the plan is finalized, the architectural, structural and electromechanical professions refine the model, in principle, $60 \%$ of the whole design depth should be completed. In this stage, the results should be reviewed and feedback by the owner, and adjusted according to the opinions. Because of the collaborative design of the whole profession, there is a connection between the different professions. Therefore, the design modification schedule of this stage will greatly improve the efficiency compared with the traditional design method.

At this stage, combined with the current status of the design industry professional design project that is not familiar with BIM design, a new type of work, BIM consultant engineer, was specifically proposed. The engineer needs to take into account the professional expertise and familiarity with BIM's design philosophy. The BIM consultant engineers get involved in the design phase, they mainly perform relevant analysis and statistics on the functional space of design drawings, digital simulation of ground transportation, and focus on the evacuation simulation of firefighting people flow and the simulation status of internal pedestrians' motion patterns. Through simulation and analysis of ventilation and sunshine performance, through analysis, they found defects in the design drawings, proposed amendments to the various professional, and brought together professional engineers to further deepen the design program. At the same time, they combined with the building model to analyze the information of main control material and construction equipment. Based on the analysis of the above aspects, the design results of this stage mainly focus on the coordination and optimization of the interior space, function and structure system of the building and the electromechanical system. At the same time, the cost of construction is controlled and visual digital simulation is provided, which makes the design more intuitive.

\subsection{Construction documents design phase}

In the construction drawing design phase, the architectural, structural and electromechanical professions have entered a comprehensive design deepening stage. While carrying out further in-depth exchanges with the owner, BIM consultant engineers combined information required 
during the construction phase to input information, perform statistics on the amount of work, conduct check analysis of the tender list, and establish a establish a complete multi-professional model visualization.

\section{BIM Model Standard}

Due to the large number of professional software involved in digital design, in order to achieve the same and exchange of internal data, and in the light of the actual situation in the current market, the following criteria are recommended for configuration:

1) Architectural, structural, and electromechanical professionals use the Autodesk Revit series of software, and the resulting file format is *.vit;

2) The building form surface and geometric positioning parameters as well as the curtain wall professional design use Rhino, Catia software, the result file format is *.3dm and *.CATProduct;

Steel structure professionals use Tekla Structures and Revit series software;

The naming rules for model files are as follows:

(1) Working Model Naming: 【Project Number】+【-】+【Design Company 】+【-】+ 【Professional Code】+【-】+【System Alphabetical Coding】+【-】+【Positioning Number】

(2) Integrated Model Naming: 【Project Number】+【-】+【Area Number】+【-】+【System Digital Coding 】+【-】+【System English Name】

(3) Component Model Naming: 【Project Number】+【-】+【Design Company 】+【-】+ 【Location】+【-】+【System Code】+【-】+【Build Type Description 】+【-】+【Build Dimension Description】+【-】+【Component Number】

(4) Type naming rules: If there's a professional number, such as the number of doors and windows, room number, etc., use the professional number directly type name;

If there are no professional numbers, such as ramps, handrails, etc., use the same format as the family name.

There are still many standards and contents for architectural design information models. According to the "Notice of the Ministry of Housing and Urban-Rural Development on Printing and Distributing the Plans for the Establishment and Revision of the Standards for Engineering Construction in 2015” from Ministry of Housing and Urban-Rural Development, there is still a lot of work to do in this area. However, it can be believed that, with the attention of all levels of departments and the continuous improvement of social acceptance and recognition, the use of BIM technology in the process of architectural design and construction has become increasingly popular. It can be foreseen that BIM technology will become the operating system for the construction industry, and more and more job assignments will be completed on BIM-based systems. Work will become more efficient with higher quality, and the results of work can be stored, searchable, with function of computable analysis, and shared collaboration.

\section{References}

[1] Chen Yuan, Li Hongxin. Theoretical Framework of BIM Collaborative Work Platform Based on Mobile Computing [J]. Construction Technology, 2015 (18).

[2] Wang Zhenhua. On Design Quality Control of Shanghai Center Tower [J]. Water Supply and Drainage, 2015 (02).

[3] Zhou Shuang, Huang Zhenhua. Analysis and Research on the Application Status of BIM in China [J]. Information Technology of Civil Construction Engineering, 2014 (04).

[4] He Guanpei. Analysis of Application Technology Route of BIM in Construction Enterprises [J]. Journal of Engineering Management, 2014 (02). 